\title{
Revista Colombiana de

\section{Endocarditis infecciosa derecha como primera manifestación de leucemia linfoblástica aguda en niños}

\section{Wilfrido Coronell-Rodríguez ${ }^{a, *}$, Cindy Arteta-Acosta ${ }^{b}$, María Teresa Rubio-Sotomayor $^{c}$, María Alejandra Suárez-Fuentes ${ }^{\mathrm{d}}$, Luis Gabriel Vinasco-Sánchez ${ }^{\mathrm{a}, \mathrm{e}}$ y Ángel Castro-Dager ${ }^{f}$}

\author{
a Pediatra Infectólogo, PhD Medicina Tropical. Docente Universidad de Cartagena, Cartagena, Colombia \\ ${ }^{\mathrm{b}}$ Médico, Universidad de Cartagena, Epidemióloga, Universidad del Norte, Cartagena, Colombia \\ c Cardióloga Pediatra, Universidad de Buenos Aires, Universidad de Cartagena, Cartagena, Colombia \\ d Infectología pediátrica. Universidad El Bosque, Bogotá, Colombia \\ e Infectología pediátrica. Universidad del Valle, Cali, Colombia \\ f Hemato-oncólogo Universidad Nacional. Docente Universidad de Cartagena, Cartagena, Colombia
}

Recibido el 25 de noviembre de 2016; aceptado el 10 de mayo de 2017

Disponible en Internet el 23 de agosto de 2017

\author{
PALABRAS CLAVE \\ Endocarditis; \\ Pediatría; \\ Antibióticos
}

\begin{abstract}
Resumen
Introducción: La endocarditis infecciosa del lado derecho representa el 5 al $10 \%$ de los casos de endocarditis infecciosa en adultos, menos frecuente en niños.

Serie de casos: Caso 1: Niña de 12 años de edad con fiebre y antecedentes de trauma en cadera izquierda por caída. Desarrolló dificultad respiratoria, choque, neumonía bilateral y celulitis en cadera izquierda. Los hemocultivos fueron positivos para Staphylococcus aureus resistente a meticilina (SARM), en tanto que el ecocardiograma mostró vegetación en la válvula tricúspide. Por bicitopenia continua se le realizó aspiración de médula ósea diagnosticándose leucemia linfocítica aguda. Recibió vancomicina durante seis semanas y posteriormente inició quimioterapia.

Caso 2: Niña de 5 años de edad, con fiebre, dificultad respiratoria, reacción leucemoide y bicitopenia, desarrolló choque con neumonía bilateral y hepatoesplenomegalia, hemocultivos positivos para SARM. El ecocardiograma mostró vegetación en válvula tricúspide y se realizó aspiración de médula ósea diagnosticándose leucemia linfocítica aguda; falleció.

Conclusión: Se reporta la concomitancia de endocarditis infecciosa derecha por SARM en pacientes con leucemia linfocítica aguda, patología con baja incidencia descrita.

(c) 2017 Sociedad Colombiana de Cardiología y Cirugía Cardiovascular. Publicado por Elsevier España, S.L.U. Este es un artículo Open Access bajo la licencia CC BY-NC-ND (http:// creativecommons.org/licenses/by-nc-nd/4.0/).
\end{abstract}

\footnotetext{
* Autor para correspondencia.

Correo electrónico: willsantiagoisabella@gmail.com (W. Coronell-Rodríguez).
} 


\section{KEYWORDS}

Endocarditis;

Paediatrics;

Antibiotics
Right-sided infective endocarditis as a first manifestation of acute lymphoblastic leukaemia in children

\begin{abstract}
Introduction: Right-sided infective endocarditis, represents from 5\% to $10 \%$ of infective endocarditis in adults, which is less frequent in children.

Case Series: Case 1: A 12 year-old girl with fever and a history a left hip injury due to a fall. She developed breathing difficulties, shock, bilateral pneumonia, and cellulitis in the left hip. The blood cultures were positive for methicillin resistant Staphylococcus aureus (MRSA), as such that the echocardiogram showed growth in the tricuspid valve. Due to persistent bicytopenia, a bone marrow aspirate was performed, with acute lymphocytic leukaemia being diagnosed. She received vancomycin for six weeks and chemotherapy was subsequently started.

Case 2: A 5 year-old girl, with fever, breathing difficulties, a leukemoid reaction, and bicytopenia, developed shock with bilateral pneumonia, and hepato-splenomegaly, as well as positive blood cultures for MRSA. The echocardiogram showed growth in the tricuspid valve, and the bone marrow aspirate performed helped diagnose an acute lymphocytic leukaemia. The patient died.

Conclusion: Right-sided infective endocarditis, concomitant with MRSA, is reported in patients with acute lymphocytic leukaemia, a disease with a low reported incidence.

(C) 2017 Sociedad Colombiana de Cardiología y Cirugía Cardiovascular. Published by Elsevier España, S.L.U. This is an open access article under the CC BY-NC-ND license (http:// creativecommons.org/licenses/by-nc-nd/4.0/).
\end{abstract}

\section{Introducción}

La endocarditis infecciosa es una entidad de difícil diagnóstico, la cual, a pesar de una terapia médica y/o intervención quirúrgica apropiada ${ }^{1}$, permanece como una de las condiciones clínicas de mayor morbilidad y mortalidad en la medicina del siglo XXI, alcanzando cifras entre $15-22 \%$ en pacientes hospitalizados y del $40 \%$ a los 5 años $^{1-3}$. Se describen dos casos de pacientes pediátricos que presentaron endocarditis infecciosa por Staphylococcus aureus resistente a meticilina (SARM) como primera manifestación de leucemia linfoblástica aguda.

\section{Caso 1}

Paciente femenina de 12 años de edad, con cuadro clínico de una semana de evolución, consistente en caída desde su propia altura con trauma en cadera izquierda acompañado de edema, tumefacción dolorosa, malestar general y fiebre cuantificada en $39^{\circ} \mathrm{C}$, por lo cual consultó al servicio de urgencias. A su ingreso se evidenció distrés respiratorio, deshidratación, somnolencia e hipotensión, por lo cual se derivó a la unidad de cuidados intensivos pediátrica. Al examen físico se evidenció en piel de muslo izquierdo celulitis con lesiones vesículo-pustulosas en miembros inferiores y superiores, y hepatomegalia. Recibió inotrópicos para manejo de la hipotensión severa. El reporte de hemocultivos fue positivo para SARM, de modo que se inició búsqueda de posibles focos de siembra del microorganismo. En vista de su pobre evolución clínica y bacteriemia, se encontró neumonía complicada con derrame bilateral, la cual ameritó toracostomía. El líquido pleural obtenido fue sembrado en botellas de hemocultivos aislándose nuevamente el SARM. Se realizó ecocardiograma doppler color transtorácico (ETT) observándose vegetación en la válvula tricúspide sin regurgitación, confirmando el diágnóstico de endocarditis infecciosa derecha (fig. 1). Se instauró tratamiento con vancomicina por seis semanas. Durante la evolución hospitalaria del proceso infeccioso (bacteriemia, infección de piel y tejidos blandos, neumonía complicada con derrame pleural y endocarditis infecciosa derecha) no tuvo la respuesta medular esperada a la infección, dado por leucopenia con neutropenia y anemia severa (hemoglobina $4,8 \mathrm{~g} / \mathrm{dl}$ ) persistentes, por lo cual se sospechó una alteración de la inmunidad celular; dentro de los estudios para confirmarlo, se realizó aspirado de médula ósea diagnosticándose leucemia linfoblástica aguda. Posterior al control del proceso infeccioso, se inició quimioterapia. Se reportó recuperación satisfactoria de sus patologías, y en la actualidad está en seguimiento ambulatorio con Cardiología pediátrica y Hemato-oncología pediátrica, en remisión completa de la enfermedad.

\section{Caso 2}

Paciente femenina de 5 años de edad, quien consultó por cuadro clínico de dos días de evolución consistente en dificultad respiratoria progresiva, fiebre cuantificada en $39^{\circ} \mathrm{C}$, adenopatías cervicales y axilares, inestabilidad hemodinámica, requerimiento de ventilación mecánica invasiva y soporte inotrópico. Los paraclínicos mostraron leucocitosis ( 37.340 células, neutrófilos $38 \%$, blastocitos $78 \%$ y hemoglobina $5,7 \mathrm{~g} / \mathrm{dl}$, células inmaduras), y hemocultivos positivos para SARM, por lo que se solicitó ecocardiograma que mostró vegetación perianular en válvula tricúspide con insuficiencia ligera y derrame pericárdico (fig. 2). Se realizó aspirado medular evidenciándose leucemia linfoblástica aguda. Se inició manejo antibiótico con vancomicina, pero ante 

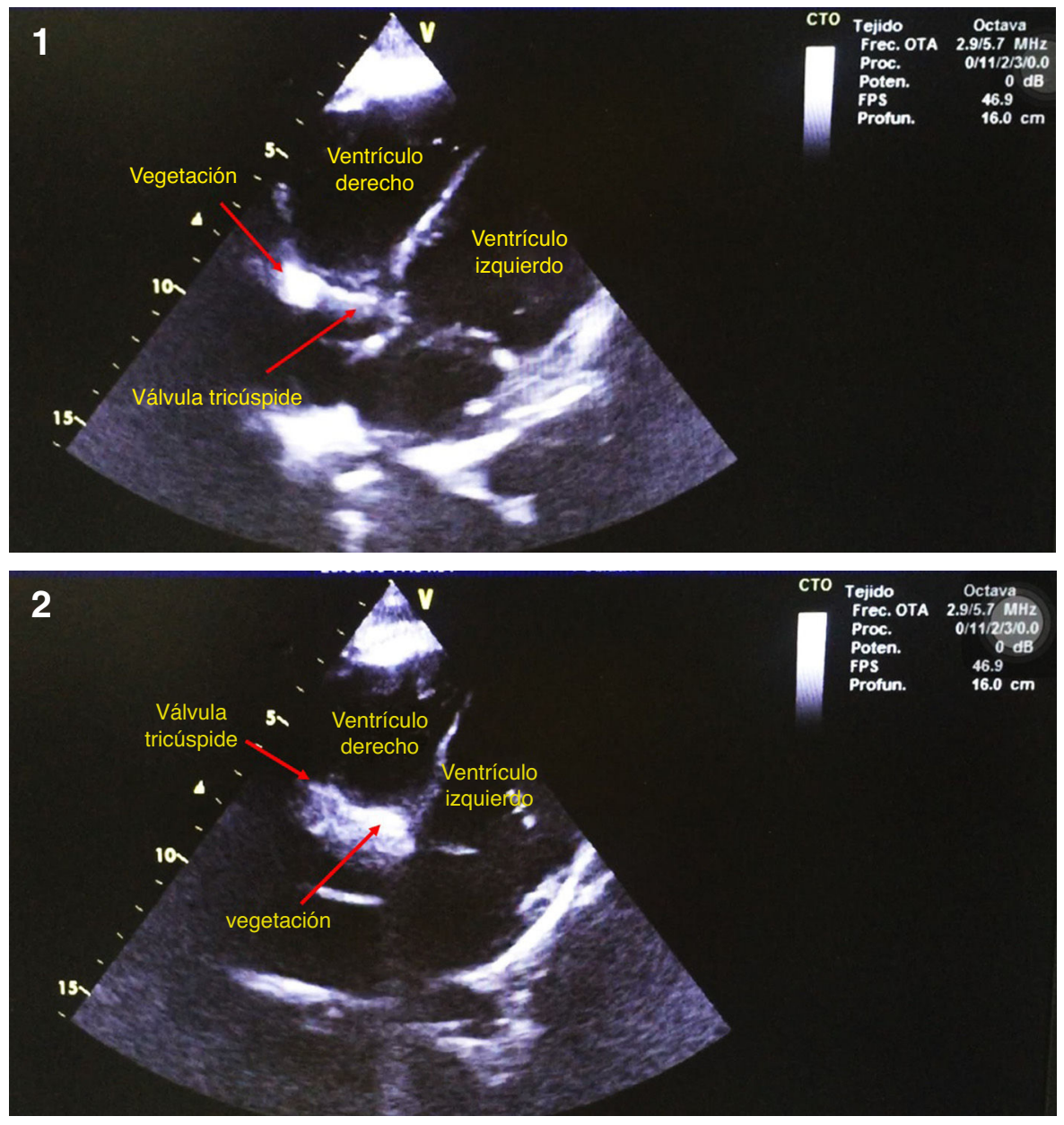

Figuras 1 y 2 Vista cuatro cámaras apical donde se aprecia imagen de zona refringente en región perianular de válvula tricúspide, en paciente con hemocultivos positivos para S. aureus y debut de leucemia.

persistencia de cuadro febril, con hemocultivos de control positivos y niveles séricos de vancomicina subterapéuticos, se cambió antibioticoterapia a daptomicina. La paciente falleció.

\section{Discusión}

La endocarditis infecciosa es poco frecuente en pacientes pediátricos; sin embargo, la literatura disponible muestra un aumento de la incidencia en dicho grupo poblacional ${ }^{1,3}$. Factores de riesgo clásicos para desarrollar endocarditis infecciosa como: enfermedad reumática, cardiopatía congénita (anormalidades de la válvula aortica, defectos septales ventriculares y tetralogía de Fallot), cirugía correctiva o paliativa de defectos cardiacos cianosantes con o sin injertos vasculares, parches o válvulas protésicas se han vuelto menos frecuentes en países desarrollados ${ }^{3}$. Sumado a los factores de riesgo descritos, la endocarditis infecciosa puede ocurrir hasta en un $56 \%$ de pacientes sin anormalidades cardíacas preexistentes, usualmente asociada a uso de catéter venoso central, drogas intravenosas y diabetes mellitus $^{1,4}$. Estudios recientes muestran una etiología cambiante en pacientes sin los antecedentes descritos antes ${ }^{5}$.
La etiología de la endocarditis infecciosa puede ser variada; predominan las bacterias grampositivas como Streptococcus viridans, Staphylococcus aureus y coagulasa negativo (asociados a accesos vasculares y material protésico), Streptococcus $\beta$ hemolítico y enterococo ${ }^{3,6}$. De forma menos común se encuentran bacterias del grupo HACEK (Haemophilus parainfluenzae, Haemophilus aphrophilus, Haemophilus influenzae, Actinobacilus actinomicetemcomitans, Cardiobacterium hominis, Eikenella corrodens, Kingella kingae, Kingella dentrificans) y hongos como cándida y aspergillus, principalmente en neonatos y usuarios crónicos de catéteres ${ }^{3,6}$.

La incidencia de endocarditis infecciosa por Staphylococcus aureus en pacientes con enfermedad hemato-oncológica se reporta en un $0,4 \%^{7-9}$. Esta incidencia es menor si se compara con la de pacientes sin enfermedad hemato-oncológica $(3,1 \%-5,4 \% \text { por año })^{7}$. Al respecto, Espersen et al. ${ }^{7}$ sugieren que esta menor incidencia puede estar dada por el inicio temprano de antibióticos de amplio espectro en este grupo de pacientes debido a su estrecha y constante observación clínica; además, dado que en estos pacientes hay un bajo recuento de plaquetas de forma frecuente, este hecho podría estar relacionado con la baja frecuencia de endocarditis infecciosa en la leucemia ${ }^{10}$; por tanto, la posibilidad de formación de vegetaciones durante la 
Tabla 1 Manifestaciones clínicas comunes de la endocarditis infecciosa ${ }^{25}$

\begin{tabular}{lc}
\hline Manifestaciones clínicas & Frecuencia (\%) \\
\hline Fiebre & $75-100$ \\
Malestar & $50-75$ \\
Anorexia & $25-50$ \\
Falla cardíaca & $25-50$ \\
Artralgia & $17-50$ \\
Esplenomegalia & $50-75$ \\
Fenómeno embólico & $25-50$ \\
Soplo (nuevo o cambiante) & $21-50$ \\
Petequias & $21-50$ \\
\hline
\end{tabular}

Adaptada de: Levasseur S, Saiman L. Endocarditis and other intravascular infections ${ }^{25}$.

bacteriemia puede ser prevenida por su trombocitopenia de base $^{7}$.

Aunque la patogénesis de la endocarditis infecciosa es compleja, puede explicarse por dos eventos principales como el desgaste o daño de la superficie endotelial que predispone a la colonización del tejido cardiaco por patógenos y la expresión de adhesinas por dichos patógenos, que funcionan como factores de virulencia y les confiere la cualidad de invadir y propagarse a través de los tejidos ${ }^{3}$.

El mecanismo por el cual el endotelio cardiaco puede verse alterado es variado y va desde turbulencias ocasionadas por flujos anómalos a través de válvulas estenóticas o insuficientes, hasta daño directo ocasionado por dispositivos que llegan a estar en contacto con dichas superficies, como catéteres intravenosos o dispositivos cardiacos implantables ${ }^{3}$.

Posterior a la alteración endotelial se desarrolla una respuesta del paciente, dada por depósitos de fibrina y plaquetas, que intentan reparar el daño endotelial subyacente, constituyendo lo que se denomina "endocarditis trombótica no bacteriana', nicho ideal para el depósito del posible agente infeccioso ${ }^{2,3}$.

La llegada del microorganismo al lugar de la disrupción endotelial puede verse favorecida por diferentes mecanismos entre los que se encuentran la inoculación directa al insertar un dispositivo intracardiaco o intravascular y la disrupción de superficies mucosas como son las del tracto gastrointestinal, genitourinario y orofaringe, esta última en actividades diarias como cepillarse los dientes y masticar alimentos, causantes de bacteriemias transitorias ${ }^{2}$.

Es así como un patógeno con la facultad de adherirse a diferentes superficies, entra en contacto con un lugar estructuralmente alterado lo cual favorece el desarrollo posterior de la endocarditis infecciosa.

La presentación clínica de endocarditis infecciosa en pediatría puede ser clasificada como subaguda o aguda. La primera típicamente se manifiesta con síntomas por varias semanas, en tanto que la aguda generalmente se presenta como una enfermedad rápidamente progresiva ${ }^{2}$. Las manifestaciones clínicas más comunes se enumeran en la tabla 1.

El diagnóstico requiere alta sospecha clínica, además de una adecuada anamnesis y examen físico, dada la inespecificidad de los síntomas. El estándar de oro para el diagnóstico siguen siendo los criterios de DUKE (tabla 2), los cuales
Tabla 2 Criterios modificados de Duke para el diagnóstico de endocarditis infecciosa ${ }^{17}$

\section{Criterios patológicos}

1. Microorganismos aislados en cultivos o prueba histológica en una vegetación, vegetación embolizada o absceso intracardiaco; o

2. Lesiones patológicas (vegetación o absceso intracardiaco) con endocarditis activa confirmada por prueba histológica.

\section{Criterios patológicos}

3. Microorganismos aislados en cultivos o prueba histológica en una vegetación, vegetación embolizada o absceso intracardiaco; 0

4. Lesiones patológicas (vegetación o absceso intracardiaco) con endocarditis activa confirmada por prueba histológica.

Criterios clínicos: dos criterios mayores, uno mayor y tres criterios menores, o cinco criterios menores.

\section{Criterios mayores}

1. Hemocultivos positivos para endocarditis infecciosa

a. Microorganismos típicos para endocarditis infecciosa de dos hemocultivos diferentes:

i. Streptococco Viridans

ii. Streptococco bovis

iii. Grupo AAECK

iv. Staphylococcus aureus

v. Enterococco adquirido en comunidad (sin foco primario)

b. Microorganismos compatibles con endocarditis infecciosa en hemocultivos con resultados persistentemente positivos si:

i. Al menos dos resultados positivos en hemocultivos separados más de 12 horas.

ii. Tres o más de cuatro hemocultivos

c. Uno solo hemocultivo para Coxiella burnetti o títulos de IgG mayores a 1:800

2. Evidencia de compromiso endocárdico por ecocardiograma positivo para endocarditis infecciosa, definido como:

a. Masa intracardiaca oscilante sobre una válvula o estructuras de soporte en el camino del jet regurgitante o en un material implatado.

b. Absceso

c. Nueva dehiscencia parcial de válvula protésica

d. Nueva regurgitación valvular (empeoramiento o cambio en murmullo preexistente no suficiente)

\section{Criterios menores}

1. Condición cardiaca predisponente o abuso de drogas intravenosas

2. Fiebre: temperatura $100,4^{\circ} \mathrm{F}$ (mayor o igual a $38^{\circ} \mathrm{C}$ )

3. Fenómeno vascular: embolia arterial mayor, infartos pulmonares sépticos, aneurismas micóticos, hemorragias intracraneales, lesiones de Janeway

4. Fenómenos inmunológicos: glomerulonefritis, nódulos de Osler, manchas de Roth, factor reumatoide

Evidencia microbiológica: hemocultivos positivos, pero que no pertenezcan a los criterios mayores o evidencia serológica de infección activa con organismos compatibles para endocarditis infecciosa 
Tabla 2 (continuación)

\section{Posible endocarditis infecciosa}

a) Un criterio mayor y un criterio menor

b) Tres criterios menores

Descartada endocarditis infecciosa

1. Manifestaciones de endocarditis presentes

en diagnósticos alternativos

2. Resolución de las manifestaciones de endocarditis

con terapia antibiótica menor a cuatro días

3. No evidencia patológica de endocarditis infecciosa en

cirugía o autopsia con terapia antibiótica menor a cuatro

días

No completa todos los criterios mencionados

Modificada de: Li JS, Sexton DJ, Mick N, Nettles R, Fowler VG, Ryan T, et al. Proposed Modifications to the Duke criteria for the diagnosis of infective endocarditis. Clin Infect Dis. 2000;30(4):633-8.

fueron modificados en el año 2002 para incluir en ellos los hallazgos ecocardiográficos ${ }^{11}$.

Mención especial merece la endocarditis infecciosa derecha, la cual es una entidad clínica rara que ocurre entre el 5 y $10 \%$ de todos los casos de endocarditis infecciosa ${ }^{12,13}$, siendo más frecuente en pacientes con historia de abuso de drogas intravenosas o en aquellos con dispositivos intravasculares. La estructura que más se afecta es la válvula tricúspide, siendo rara la afección aislada de la válvula pulmonar. La menor incidencia respecto a la endocarditis del lado izquierdo se debe a una presentación menos frecuente de cardiopatías congénitas del lado derecho, baja presión en las válvulas de dicho lado y menor saturación de oxígeno ${ }^{14}$.

La endocarditis infecciosa derecha se divide en los siguientes grupos clínicos, de acuerdo con los antecedentes:

1. Endocarditis del lado izquierdo.

2. Uso de drogas endovenosas o dispositivos cardiacos.

3. Grupo de los "NO" (no endocarditis izquierda, no uso de drogas, no dispositivos cardiacos) ${ }^{13,15,16}$.

Los agentes etiológicos de la endocarditis infecciosa derecha no difieren de los causantes de la endocarditis infecciosa $^{6,16}$. Una característica clínica importante es la presencia de émbolos hacia la circulación pulmonar a diferencia de la presentación en la endocarditis infecciosa del lado izquierdo en la cual puede ocurrir embolización periférica $^{6,16}$. Además, se puede encontrar síndrome tricuspídeo, descrito por Nandakumar y Raju, caracterizado por fiebre prolongada, eventos respiratorios recurrentes como neumonía (frecuentemente asintomática), embolia pulmonar, anemia y hematuria microscópica ${ }^{12,13,17}$.

La endocarditis infecciosa es un síndrome complejo, cuyo diagnóstico requiere microbiología especializada, imágenes y exámenes de laboratorio y biomarcadores ${ }^{2}$.

Los hemocultivos continúan siendo la piedra angular en el diagnóstico de esta entidad, siendo positivos entre un $70-90 \%$ de los $\operatorname{casos}^{3}$, permitiendo la identificación del germen $^{1,2,15}$. Es importante obtener al menos tres hemocultivos tomados en tiempos diferentes dependiendo de la estabilidad del paciente; si se encuentra inestable, deberán ser tomados en un corto periodo de tiempo (una a dos horas) y en caso de estabilidad, pueden ser tomados en mayor tiempo (6 a 8 horas) ) $^{1,2,18,19}$. Las muestras para los cultivos deben obtenerse de una vena periférica y en caso de que el paciente tenga un catéter venoso central, una de las muestras debe ser tomada de este $\mathrm{e}^{15}$. Es necesario tomar volúmenes adecuados de sangre ${ }^{2}$ : en neonatos y lactantes de $1 \mathrm{a} 3 \mathrm{ml}$, en niños mayores de $5-7 \mathrm{ml}$ y en adolescentes y adultos $10 \mathrm{ml}^{2,3,15}$.

Cuando en los hemocultivos se logra aislar el microorganismo, se deben tomar hemocultivos de control a las 2448 horas para evaluar la efectividad del tratamiento, hasta que se obtengan cultivos negativos ${ }^{18,19}$. Entre un 10$30 \%$ de los hemocultivos pueden ser negativos debido a inicio de terapia antibiótica previa a la toma de cultivos, etiología diferente a la bacteriana o presencia de microorganismos fastidiosos (Coxiella burnetii, Bartonella spp., Mycoplasma pneumoniae, Brucella spp. y Legionella pneumophila, Abiotrophia y Granulicatella), por lo que ante esta situación se sugiere realizar pruebas serológicas o reacción en cadena de la polimerasa ${ }^{1,3,15,20}$.

Se han evaluado algunos biomarcadores con el fin de estratificar la gravedad de la endocarditis infecciosa: leucocitosis/leucopenia, número de células inmaduras mayor al $10 \%$, aumento de proteína $C$ reactiva, procalcitonina y velocidad de sedimentación globular. De igual forma, marcadores de disfunción orgánica (lactato, bilirrubinas, plaquetas y creatinina sérica) pueden encontrarse alterados $^{15}$. Los niveles de algunos mediadores inflamatorios como hipocomplementemia, factor reumatoide positivo y hematuria, avalan pero no hacen parte de los criterios diagnósticos de endocarditis infecciosa ${ }^{2}$, sobretodo en presencia de vasculitis ${ }^{15}$. Por otro lado, el aumento del péptido- $B$ natriurético y niveles de troponina pueden indicar lesión cardíaca ${ }^{2}$.

En el diagnóstico de endocarditis infecciosa derecha, al igual que la endocarditis infecciosa, los criterios de DUKE siguen siendo el estándar de oro ${ }^{11}$. Sin embargo, se ha propuesto la adición del síndrome tricuspídeo ${ }^{21}$, para aumentar su sensibilidad y especificidad en este escenario ${ }^{21}$.

El ecocardiograma doppler color (ECO) es una herramienta importante para el diagnóstico y manejo de la endocarditis infecciosa ${ }^{1,4,6}$; debe hacerse de manera oportuna en todos los casos en que se sospeche esta patología1,22,23. En la población pediátrica, el ETT, si hay buena ventana ecocardiográfica, es ideal para identificar y hacer seguimiento de una masa intracardiaca oscilante, absceso perianular, dehiscencia parcial de una prótesis o una nueva insuficiencia valvular, además de algunos hallazgos de endocarditis infecciosa derecha como vegetaciones en la válvula tricúspide, anormalidades en el tracto de salida del ventrículo derecho y disfunción de la válvula tricúspide ${ }^{1,2,4,6}$; en caso de que la ventana ecocardiográfica sea inadecuada o coexistan condiciones como enfermedad pulmonar obstructiva crónica, antecedente de cirugía de tórax u obesidad mórbida o cuando persiste la alta sospecha de endocarditis infecciosa con ETT negativo, se indica el TEE (ecocardiograma doppler color transesofágico $)^{4}$. Si la sospecha clínica persiste y el resultado del ecocardiograma no es el esperado, este debe repetirse entre 7 a 10 días posteriores ${ }^{4}$.

El principio de tratamiento en esta patología en el contexto pediátrico es similar que en adultos ${ }^{3}$, teniendo en cuenta que por su alta complejidad, el enfoque 
Tabla 3 Tratamiento antibiótico de la endocarditis infecciosa con base en aislamiento

\begin{tabular}{|c|c|c|}
\hline Germen & Elección & Alternativa \\
\hline Streptococcus sensible a penicilina & Penicilina & Vancomicina \\
\hline $\mathrm{CIM}^{*} \leq 0,1 \mu \mathrm{g} / \mathrm{ml}$ & 0 & 0 \\
\hline \multirow[t]{4}{*}{ S. viridans, S. Grupo A, B, C, D, G } & Ampicilina & Cefalosporina de primera generación \\
\hline & 0 & 0 \\
\hline & Ceftriaxona & Ceftriaxona \\
\hline & $\begin{array}{l}+ \\
\text { (aminoglucócido si hay válvula protésica } \\
\text { primeras dos semanas) }\end{array}$ & \\
\hline Streptococcus con resistencia & Penicilina (o ampicilina) & Si es Enterococcus y paciente es alérgico \\
\hline intermedia a penicilina & & a B-lactámico o tiene válvula protésica: \\
\hline $\mathrm{CIM}^{*} \geq 0,2 \mu \mathrm{g} / \mathrm{ml}$ & gentamicina & vancomicina \\
\hline incluyendo Enterococcus y S. viridans & & + \\
\hline \multirow[t]{9}{*}{ con susceptibilidad reducida } & & Gentamicina \\
\hline & & No Enterococcus: \\
\hline & & Ceftriaxona \\
\hline & & + \\
\hline & & Gentamicina \\
\hline & & $\begin{array}{l}\text { Enterococcus resistente a } \\
\text { aminoglucócido: }\end{array}$ \\
\hline & & Ampicilina \\
\hline & & + \\
\hline & & Ceftriaxona \\
\hline \multirow{3}{*}{$\begin{array}{l}\text { Enterococcus resistentes a penicilina, } \\
\text { aminoglucócido y vancomicina }\end{array}$} & Linezolid & \\
\hline & & \\
\hline & Daptomicina & \\
\hline \multirow[t]{5}{*}{ SASM $^{a}$} & Oxacilina & \\
\hline & 0 & \\
\hline & Nafcilina & \\
\hline & 0 & \\
\hline & Cefalosporina de primera generación & \\
\hline \multirow[t]{4}{*}{ SARM $^{\mathrm{b}}$} & Vancomicina & Daptomicina \\
\hline & + & \\
\hline & Gentamicina & \\
\hline & $\begin{array}{l}\text { Considere adicionar rifampicina si hay } \\
\text { válvula protésica }\end{array}$ & \\
\hline \multirow[t]{2}{*}{ Bacilos entéricos Gram (-) } & $\begin{array}{l}\text { Ceftazidime, cefepime, cefotaxime, } \\
\text { ceftriaxona }\end{array}$ & $\begin{array}{l}\text { Piperacilina tazobactam } \\
+\end{array}$ \\
\hline & $\begin{array}{l}+ \\
\text { gentamicina o amikacina o tobramicina }\end{array}$ & Gentamicina, tobramicina o amikacina \\
\hline \multirow[t]{2}{*}{ GRUPO HACEK } & $\begin{array}{l}\text { Ceftriaxona o cefotaxime o ampicilina } \\
\text { sulbactam }\end{array}$ & $\begin{array}{l}\text { Ampicilina (si hay susceptibilidad) } \\
+\end{array}$ \\
\hline & & Aminoglucócido \\
\hline
\end{tabular}

Adaptado de: Infective Endocarditis in Childhood: 2015 Update $^{3}$.

* CIM: concentración inhibitoria mínima.

a Staphylococcus aureus sensible a meticilina.

b Staphylococcus aureus resistente a meticilina.

terapéutico deberá estar a cargo de un grupo multidisciplinario que cuente, entre otros, con Cardiología pediátrica, Pediatría, Infectología pediátrica y Cirugía cardiovascular ${ }^{2}$.

La terapia antimicrobiana empírica comienza temprano con antibióticos que tengan actividad bactericida y concentraciones séricas adecuadas ${ }^{6}$. Su elección depende de la edad del paciente, el estado hemodinámico, el estatus cardiovascular de base, la forma de adquisición (comunidad o asociada al cuidado de la salud), el tiempo de evolución (aguda, subaguda o crónica), el uso previo de antibióticos y los microorganismos probables causantes de la infección ${ }^{6}$. Una vez identificado el patógeno, la terapia se guiará por las pruebas de susceptibilidad antimicrobiana ${ }^{6}$.

La farmacodinámica de los antibióticos en esta patología puede verse alterada debido a que en las vegetaciones se puede encontrar una alta concentración del inóculo bacteriano, subpoblaciones bacterianas en diferentes estadios de crecimiento, formación de biofilm y fibrina y plaquetas, que limitan la entrada del antibiótico ${ }^{3,6}$. 
En general, las guías actuales indican que el manejo empírico inicial en pacientes con endocarditis infecciosa en válvula nativa, comienza con ampicilina/sulbactam más un aminoglucócido; la adición o no de vancomicina al esquema anterior está sujeta a la sospecha de infección por Staphylococcus aureus. En endocarditis infecciosa de válvula protésica el manejo empírico se hará con vancomicina + gentamicina + rifampicina. Si la endocarditis infecciosa ocurre en válvula protésica menor de un año o asociada a los cuidados de la salud, al esquema anterior se le agregará cefepime. Adicionalmente, los esquemas anteriores deberán ajustarse de acuerdo con el resultado de los hemocultivos (tabla 3) 3,6,18,20.

La duración de la terapia antimicrobiana en endocarditis infecciosa oscila entre 4 a 8 semanas a partir del momento en que se negativicen los hemocultivos, y depende de factores como el patógeno aislado (tabla 3 ) y el tipo de válvula

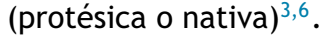

El manejo quirúrgico en la endocarditis infecciosa derecha está indicado en disfunción valvular que genera signos y síntomas de falla cardiaca, ante complicación con bloqueo cardiaco, abscesos aórticos o anulares, en infecciones por hongos u organismos altamente resistentes (enterococo vancomicinorresistente, bacilos gramnegativos multidrogorresistentes, SAMR), vegetación mayor de $10 \mathrm{~mm}$ de diámetro o aumento del tamaño de ésta pese a tratamiento antibiótico instaurado, más de un evento embólico durante las primeras dos semanas de tratamiento antibiótico y perforación o ruptura de aparato valvular $4,15,24,25$.

\section{Conclusión}

La endocarditis infecciosa derecha es una patología rara y más aún en pacientes con enfermedad hematológica maligna, en cuyo caso, el abordaje, diagnóstico y tratamiento oportunos son fundamentales para el desenlace y pronóstico de la misma.

\section{Responsabilidades éticas}

Protección de personas y animales. Los autores declaran que para esta investigación no se han realizado experimentos en seres humanos ni en animales.

Confidencialidad de los datos. Los autores declaran que han seguido los protocolos de su centro de trabajo sobre la publicación de datos de pacientes.

Derecho a la privacidad y consentimiento informado. Los autores han obtenido el consentimiento informado de los pacientes y/o sujetos referidos en el artículo. Este documento obra en poder del autor de correspondencia.

\section{Financiación}

Propia de los autores.

\section{Conflicto de intereses}

Ninguno.

\section{Consideraciones éticas}

Se obtuvo aprobación de los acudientes de las menores.

\section{Bibliografía}

1. Nishimura RA, Otto CM, Bonow RO, Carabello BA, Erwin JP, Guyton RA, et al. 2014 AHA/ACC guideline for the management of patients with valvular heart disease. J Am Coll Cardiol. 2014;63:e57-185.

2. Bragg L, Alvarez A. Endocarditis. Pediatr Rev. 2014;35:162-8.

3. Baltimore RS, Gewitz M, Baddour LM, Beerman LB, Jackson MA, Lockhart PB, et al. Infective endocarditis in childhood: 2015 update: a scientific statement from the American Heart Association. Circulation. 2015;132:1487-515.

4. Day MD, Gauvreau K, Shulman S, Newburger JW. Characteristics of Children hospitalized with infective endocarditis. Circulation. 2009;119:865-70.

5. Lee M-R, Chang S-A, Choi S-H, Lee G-Y, Kim E-K, Peck K-R, et al. Clinical features of right-sided infective endocarditis occurring in non-drug users. J Korean Med Sci. 2014;29:776.

6. Baddour LM. Infective endocarditis: diagnosis, antimicrobial therapy, and management of complications: a statement for healthcare professionals from the Committee on Rheumatic Fever, Endocarditis, and Kawasaki Disease, Council on Cardiovascular Disease in the Young, and the Councils on Clinical Cardiology, Stroke, and Cardiovascular Surgery and Anesthesia, American Heart Association: Endorsed by the Infectious Diseases Society of America. Circulation. 2005;111: e394-434.

7. Espersen F, Frimodt-Møller N, Rosdahl VT, Jessen O, Faber V, Rosendal K. Staphylococcus aureus bacteremia in patients with hematological malignancies and/or agranulocytosis. Acta Med Scand. 1987;222:465-70.

8. Belik J, Flinn G, Rivera G, Sanyal SK. Successful management of bacterial endocarditis of the mitral valve due to Staphylococcus epidermidis in an immunosuppressed host. Acta Paediatr Scand. 1980;69:731-4.

9. Srinivasan A, Seifried S, Zhu L, Srivastava DK, Flynn PM, Bankowski MJ, et al. Staphylococcus aureus bacteremia in pediatric patients with cancer. Pediatr Infect Dis J. 2010;29: 172-4.

10. Keino D, Tsuzuki Y, Mori T, Kakuage S, Nakano M, Asoh K, et al. Infective endocarditis associated with acute leukemia: Report of two cases: Endocarditis with acute leukemia. Pediatr Int. 2015;57:1017-20.

11. Thanavaro KL, Nixon JV (Ian). Endocarditis 2014: An update. Heart Lung J Acute Crit Care. 2014;43:334-7.

12. Yameogo NV, Sondo KA, Yameogo AA, Kagambega LJ, Mand DG, Kologo KJ, et al. Epidemiological and clinical features, ultrasound findings and prognosis of right-sided infective endocarditis in a teaching hospital in Ouagadougou: cardiovascular topics. Cardiovasc J Afr. 2013;24:171-3.

13. Ortiz C, López J, García H, Sevilla T, Revilla A, Vilacosta I, et al. Clinical classification and prognosis of isolated rightsided infective endocarditis: Medicine (Baltimore). 2014;93: e137.

14. Akinosoglou K, Apostolakis E, Koutsogiannis N, Leivaditis V, Gogos CA. Right-sided infective endocarditis: surgical management. Eur J Cardiothorac Surg. 2012;42:470-9.

15. Habib G, Lancellotti P, Antunes MJ, Bongiorni MG, Casalta JP, Del Zotti F, et al. 2015 ESC Guidelines for the management of infective endocarditis: The Task Force for the Management of Infective Endocarditis of the European Society of Cardiology (ESC)Endorsed by: European Association for Cardio-Thoracic 
Surgery (EACTS), the European Association of Nuclear Medicine (EANM). Eur Heart J. 2015;36:3075-128.

16. Chahoud J, Sharif Yakan A, Saad H, Kanj S. Right-sided infective endocarditis and pulmonary infiltrates: an update. Cardiol Rev. 2015:1.

17. Nandakumar R, Raju G. Isolated tricuspid valve endocarditis in nonaddicted patients: a diagnostic challenge. Am J Med Sci. 1997;314:207-12.

18. Liu C, Bayer A, Cosgrove SE, Daum RS, Fridkin SK, Gorwitz RJ, et al. Clinical Practice Guidelines by the Infectious Diseases Society of America for the treatment of methicillin-resistant Staphylococcus aureus infections in adults and children. Clin Infect Dis. 2011;52:e18-55.

19. Baron EJ, Miller JM, Weinstein MP, Richter SS, Gilligan PH, Thomson RB, et al. A Guide to utilization of the microbiology laboratory for diagnosis of infectious diseases: 2013 Recommendations by the Infectious Diseases Society of America (IDSA) and the American Society for Microbiology (ASM)a. Clin Infect Dis. 2013;57:e22-121.
20. Hoen B, Duval X. Infective endocarditis. N Engl J Med. 2013;368:1425-33.

21. Carrillo-Esper R, Rangel-Olascoaga C. Endocarditis tricuspídea. Med Int Méx. 2014;30:209-14.

22. Haldar SM, O'Gara PT. Infective endocarditis: diagnosis and management. Nat Clin Pract Cardiovasc Med. 2006;3: 310-7.

23. Bashore TM, Cabell C, Fowler V Jr. Update on infective endocarditis. Curr Probl Cardiol. 2006;31:274-352.

24. Baddour LM, Wilson WR, Bayer AS, Fowler VG, Tleyjeh IM, Rybak MJ, et al. Infective endocarditis in adults: diagnosis, antimicrobial therapy, and management of complications: a scientific statement for healthcare professionals from the American Heart Association. Circulation. 2015;132:1435-86.

25. Levasseur S, Saiman L. Endocarditis and other intravascular infections. En: Principles and Practice of Pediatric Infectious Diseases. $4^{\text {th }}$. ed. Long SS, Pickering LK, Prober CG. Saunders Elsevier; 2012. p. 256-65. 